\title{
Molecular profiling of indolent human prostate cancer: tackling technical challenges to achieve high-fidelity genome-wide data
}

\begin{abstract}
Thomas A. Dunn', Helen L. Fedor ${ }^{2}$, Angelo M. De Marzo², Jun Luo ${ }^{1}$
The contemporary problem of prostate cancer overtreatment can be partially attributed to the diagnosis of potentially indolent prostate cancers that pose low risk to aged men, and lack of sufficiently accurate risk stratification methods to reliably seek out men with indolent diseases. Since progressive acquisition and accumulation of genomic alterations, both genetic and epigenetic, is a defining feature of all human cancers at different stages of disease progression, it is hypothesized that RNA and DNA alterations characteristic of indolent prostate tumors may be different from those previously characterized in the setting of clinically significant prostate cancer. Approaches capable of detecting such alterations on a genome-wide level are the most promising. Such analysis may uncover molecular events defining early initiating stages along the natural history of prostate cancer progression, and ultimately lead to rational development of risk stratification methods for identification of men who can safely forego treatment. However, defining and characterizing indolent prostate cancer in a clinically relevant context remains a challenge, particularly when genome-wide approaches are employed to profile formalin-fixed paraffin-embedded (FFPE) tissue specimens. Here, we provide the conceptual basis underlying the importance of understanding indolent prostate cancer from molecular profiling studies, identify the key hurdles in sample acquisition and variables that affect molecular data derived from FFPE tissues, and highlight recent progresses in efforts to address these technical challenges.
\end{abstract}

Asian Journal of Andrology (2012) 14, 385-392; doi:10.1038/aja.2011.147; published online 6 February 2012

Keywords: active surveillance; formalin-fixed paraffin-embedded; indolent prostate cancer; microarray; molecular profiling; prostate cancer; prostate cancer progression; risk stratification

\section{INTRODUCTION}

It is well recognized that a substantial proportion of prostate cancer patients diagnosed in the prostate-specific antigen (PSA) era harbor clinically insignificant and potentially indolent diseases, and thus may not benefit from definitive treatments. ${ }^{1}$ Development of methods to seek out men from newly diagnosed patients with truly indolent prostate cancer, who can safely forego treatment, represents a major contemporary challenge in many Western countries. In other geographic regions where PSA testing as a screening tool is increasingly adopted in practice, prostate cancer overdiagnosis and overtreatment is also likely to become a major issue. Indolent prostate cancer in a clinically relevant context can be defined as biopsy-detected prostate tumors that, if left untreated, will not progress to clinically significant diseases during the lifetime of the patient. Unfortunately, we have very limited understanding of indolent prostate cancer. It is not clear, for example, whether indolent prostate cancer simply represents early lesions preceding the development of aggressive, clinically significant disease, or arises independently and may remain indolent, and thus will not drive the natural history of disease progression. ${ }^{2}$ Importantly, molecular profiling studies targeting small-volume, low-risk (for progression), potentially indolent prostate cancers have not been conducted systemically. Such studies would entail the establishment of an informative cohort of patients diagnosed with low-risk prostate cancer and followed up longitudinally for development of higher-risk disease, and will rely on the quality and quantity of RNA and DNA that can be retrieved from small-volume, formalin-fixed paraffin-embedded (FFPE) tissue lesions. Because of the lack of such studies, it is not known why some prostate tumors remain indolent whereas others progress to clinical malignancy. A more complete understanding of the natural history of prostate cancer progression and relevant molecular events will undoubtedly facilitate clinical development of methods for risk stratification at the time of diagnosis. This article advocates the use of molecular approaches for the discovery and development of markers defining indolent prostate cancer, and will address a number of critical issues pertinent to molecular profiling of indolent prostate cancer on the genome-wide level. When appropriate, our experiences in dealing with molecular analysis of FFPE tissues will be highlighted.

\section{LOW-RISK PROSTATE CANCER: THE DILEMMA BETWEEN CURATIVE TREATMENT AND OVERTREATMENT}

While prostate cancer is widely perceived as the most common nonskin cancer in the Western world, many of the diagnosed cancers are

${ }^{1}$ Department of Urology, Johns Hopkins University, Baltimore, MD 21287, USA and ${ }^{2}$ Department of Pathology, Johns Hopkins University, Baltimore, MD 21287, USA Correspondence: Dr. J Luo (jluo1@jhmi.edu)

Received: 15 September 2011; Revised: 13 October 2011; Accepted: 13 October 2011; Published online: 6 February 2012 
potentially clinically insignificant. For example, among the estimated 232,090 newly diagnosed prostate cancer cases in the United States of America in the year $2005,{ }^{3}$ more than $80 \%$ will likely survive for over 20 years. ${ }^{4}$ Multiple factors account for this high survival rate of newly diagnosed prostate cancer patients in the United States of America: (i) in the modern PSA era, prostate cancers are discovered early; (ii) most localized prostate cancers are inherently slow growing in nature; and (iii) effective treatments exist for clinically localized prostate cancer. On the one hand, continued development of early diagnostic methods and implementation of screening tools may help to lower prostate cancer-specific death rates, ${ }^{5}$ which happens at a frequency of one death every $17.3 \mathrm{~min}$ in the United States of America alone. ${ }^{3}$ On the other hand, overdiagnosis, which inevitably leads to overtreatment, has substantial quality of life impact on patients and the health-care system. This dilemma is further exacerbated by the well-known fact that histological prostate cancer is present in a large proportion of aged men. Based on estimates from the autopsy studies of Sakr et al. ${ }^{6}$ and Powel et al., ${ }^{7}$ prostate cancer could be found in $\sim 40 \%$ of 40 -year-old men, $50 \%$ of 50 -year-old men, $60 \%$ of 60 -year-old men and $70 \%-80 \%$ of men aged over 70 years. These incidental cancers, mostly considered indolent and thus posing very low risk to aged men, may be detected at biopsy (often triggered by elevated PSA), leading to diagnosis of prostate cancer in men that would not have been diagnosed during their lifetime if a PSA test were not used (i.e., overdiagnosis). ${ }^{8}$ This notion is supported by findings from the Prostate Cancer Prevention Trial, ${ }^{9}$ in which end-of-study biopsies identified prostate cancer in $25 \%$ of study participants in the placebo arm (mean age: 63 years), a rate four times higher than originally anticipated. Overall, these findings predict a much higher prostate cancer incidence rate following the implementation of more aggressive and effective prostate cancer screening strategies in many developing countries (e.g., China). Indiscriminate definitive treatment of screen-detected prostate cancer may help to decrease the overall prostate cancer death rate, but at a potential cost of unnecessary adverse quality of life effects and additional health care burden to many.

\section{ACTIVE SURVEILLANCE FOR THE MANAGEMENT OF PATIENTS WITH LOW-RISK PROSTATE CANCER}

Diagnostic biopsy findings, when combined with other clinical parameters, have been widely used to evaluate the risk status of newly diagnosed prostate cancers. Favorable tumor biopsy and clinical findings may be indicative of the indolent nature of detected prostate tumors, and they have been used to inform whether immediate treatment would benefit the patients in many active surveillance programs. ${ }^{1,10}$ For example, the active surveillance program at the Johns Hopkins University, started by Dr. Ballentine Carter and Dr. Jonathan Epstein in 1995, has enrolled 964 men (as of March 2011; median age: 67 years). Under this program, men suspected of harboring very lowrisk prostate cancer are offered active surveillance as an alternative to immediate curative intervention. ${ }^{1,11-16}$ Patients are followed up prospectively. The surveillance protocol defines patient eligibility criteria, includes semiannual PSA measurement with digital rectal examination, and annual 12- to 14-core surveillance biopsy.

However, implementation of active surveillance for management of low-risk prostate cancer is currently limited, largely due to the perceived risk of disease progression; most men seek definitive treatment despite meeting the entry criteria for active surveillance, fearing clinical progression and a lost window of opportunity for cure. Indeed, among patients meeting the entry criteria for active surveillance but treated with radical retropubic prostatectomy, pathological examination of radical retropubic prostatectomy specimens will confirm about $60 \%-80 \%$ very low-risk prostate cancers while upgrading a subgroup of cases (about 20\%-40\%) to higher-risk disease. ${ }^{1,11,17}$ Therefore, while active surveillance would alleviate the burden of prostate cancer overtreatment, the obvious challenge is to identify those men with truly low-risk disease, i.e., indolent prostate cancer, who can safely forego treatment.

\section{POTENTIAL ROLE OF MOLECULAR MARKERS}

If men harboring indolent prostate cancer can be identified based on their molecular profiles/markers at the time of diagnosis, informative treatment decisions can then be made such that those with indolent diseases can be safely monitored. However, our understanding of the molecular profiles in indolent prostate tumors is very limited, especially with regard to longitudinal molecular changes that are associated with developing higher-risk disease. Previous molecular profiling studies of human prostate cancer have focused on molecular alterations distinguishing cancer versus normal, or recurrent/ advanced cancer versus less advanced (but still clinically significant and high-risk) cancer. It is possible to test candidate markers derived from these studies ${ }^{18-20}$ for evaluation of their ability to differentiate indolent and aggressive prostate cancers. However, due to sampling bias toward high-risk tumors, the candidate markers signifying highrisk prostate tumors may not be directly relevant to the goal of identifying molecular alterations distinguishing indolent prostate cancer and aggressive prostate cancer. Approaches capable of detecting RNA and DNA changes on a genome-wide level directly in indolent prostate cancers would be more relevant, if the most informative profiles are to be obtained.

\section{CONCEPTUAL BASIS FOR MOLECULAR PROFILING OF INDOLENT PROSTATE CANCER}

Prostate carcinogenesis is thought to be a lengthy process, and the transition from normal prostate epithelium to clinical manifestation of cancer may take decades. ${ }^{21}$ Moreover, some initiated foci of prostate cancer may remain indolent, while others progress to clinical malignancy due to differences in molecular profiles. Since progressive acquisition and accumulation of genomic alterations, both genetic and epigenetic, is a defining feature of all human cancers at different stages of disease progression, ${ }^{22}$ we propose that RNA and DNA alterations characteristic of indolent prostate tumors may be different from those detected in previous studies utilizing predominantly largevolume, clinically significant tumors.

While a temporal order of molecular alterations clearly takes place during the course of cancer progression, previous microarray studies have suggested a remarkably stable expression signature over the course of cancer progression. For example, paired pre- and posttreatment cancer tissues often cluster together based on patient identity rather than treatment status, and cancers taken from primary and distant sites from the same patient are more similar than those from the same site but different patients. ${ }^{23}$ It is therefore likely that the 'progression signature' is present in the target tissue even before the manifestation of actual progression. A recent study suggests that prostate cancer progression and metastasis may be driven by clonally derived lesions with defined molecular alterations. ${ }^{24}$ Indeed, a consistent observation from multiple earlier expression profiling studies is that it is possible to predict the past (heritable mutation status) and future (clinical course of progression) events associated with cancer initiation and progression. For example, gene expression profiles of adenocarcinoma metastases of multiple tumor types were compared 
to unmatched primary adenocarcinomas. ${ }^{25}$ This study found that solid tumors carrying the gene-expression signature resembling that of metastases were most likely to be associated with metastasis and poor clinical outcome, thereby suggesting that the metastatic potential of primary tumors is encoded in the expression profile of the primary tumor. In another study, distinct expression profiles were identified among breast tumors harboring inherited BRCA1 and BRCA2 mutations and sporadic breast cancers, and class prediction methods were used to predict the mutation status accurately. ${ }^{26}$ The results suggested that the distinctive expression profile correlated with heritable mutations or early molecular alterations is likely to be retained, at least partially, during cancer progression. In other words, genetic and environmental factors that ultimately determine the biological and clinical behavior of different types of cancers may be defined at a stage well before their clinical manifestation. Particularly relevant to profiling of indolent prostate cancer, tumors that remain indolent during the course of active surveillance may harbor an 'indolent signature', while those progressing to clinical malignancy may harbor clonally different lesions/cells with the 'aggressive signature'. Genetic, epigenetic or gene expression changes that define such signatures have yet to be fully explored and characterized in the setting of newly diagnosed prostate cancer.

\section{TECHNICAL HURDLES IN GENOME-WIDE ANALYSIS OF INDOLENT PROSTATE CANCER}

Our current lack of understanding of the molecular signature defining indolent prostate cancer is directly related to a number of technical hurdles inherent in the analysis of these tumors. By definition, indolent prostate tumors are small volume in sizes, presenting several unique technical hurdles pertaining to the procurement of high-quality RNA and DNA from histologically defined lesions. First, these tumors do not qualify for harvesting as frozen tissues, because accurate diagnosis (by pathological evaluation) of such tumors would be compromised if frozen tissues were harvested. Second, to avoid contamination by the mostly normal tissues adjacent to the small tumor foci, histological foci consistent with the pathological definition of indolent tumors need to be separated from the adjacent normal-appearing tissues. Successful procurement of tissue lesions for analysis will involve concerted efforts in case identification and selection, as well as separation of lesions of interest (e.g., by laser capture microdissection (LCM)). Third, since FFPE tissues are the main source of such specimens, a general strategy involving the use of FFPE tissues, coupled with LCM, DNA and RNA extraction, DNA and RNA amplification, and rigorous quality control, needs to be implemented. In spite of these challenges, the technical hurdles are not insurmountable, as demonstrated by recent advances in relevant areas. Recent efforts in tackling technical barriers to achieve high-fidelity molecular data from FFPE specimens ${ }^{27-32}$ have lead to the following conclusions:

1. Pathological lesions that depend on the FFPE procedure for accurate identification can be effectively profiled using recently processed specimens.

2. Prospective molecular archiving involving long-term storage in conditions compatible with RNA and DNA preservation can be implemented to allow molecular studies in the future.

3. Optimal duration of fixation needs to be determined in a clinical setting and may lead to further improvement.

In the remaining sections of this article, we will highlight these advances and focus on our own experience in efforts to achieve high-fidelity genome-wide molecular data from small-volume FFPE prostate tissue lesions.

\section{POTENTIAL VARIABLES AFFECTING FIDELITY OF MOLECULAR DATA DERIVED FROM FFPE TISSUE}

In genome-wide studies involving prostate cancer, a major issue is that tissues most suitable for molecular profiling studies do not capture the spectrum of the disease in a contemporary patient population. Molecular studies involving DNA and RNA analysis often require high-quality fresh-frozen specimens from which high quality data can be readily obtained. Frozen prostate cancer tissues are usually limited in the number of cases represented and also inevitably biased toward large-volume tumors. FFPE specimens are the standard diagnostic material compatible with the clinical setting. The FFPE procedure also preserves tissue morphology and may facilitate studies investigating molecular correlates of defined lesions that can not be effectively identified in frozen tissues. In addition, FFPE tissues are available in large quantities suitable for large-scale studies in more representative patient populations. The quality and quantity of RNA and DNA extracted from FFPE tissues, however, is often inferior when compared to frozen tissues. It is important to dissect the various variables involved in the FFPE procedure that may adversely affect the fidelity of molecular data derived from FFPE tissues. Key variables are summarized in Figure 1, and included those introduced before and after diagnostic evaluation of the FFPE specimen. It is anticipated that by addressing each of the key variables, we will be able to increasingly improve the utility of FFPE specimens for molecular profiling studies, and turn the FFPE tissues from a 'trash mine' to 'gold mine' (Figure 1).

\section{QUALITY OF RNA EXTRACTED FROM RECENTLY PROCESSED FFPE SPECIMENS}

Our general experience is that RNA extracted from aged FFPE tissues are usually of poor quality and low yield (unpublished data, Jun Luo, 2008). Our recent efforts ${ }^{32}$ focused on recently processed FFPE tissues (within 3 months between FFPE procedure and tissue isolation). FFPE tissues in our institution are mainly prepared by using one of the three methods shown in Figure 2, reproduced from a recent publication. ${ }^{32}$ These methods are standard tissue-harvesting procedure (harvested), a special tissue-harvesting procedure for specimens with relatively small areas of tumor (pinned), and whole prostate formalin injection and microwaving (injected). We first performed experiments to assess whether current tissue harvesting and fixation methods employed in our institution compromise subsequent RNA extraction. Figure 2, reproduced from a recent publication, ${ }^{32}$ shows the consistent high quality of RNA derived from FFPE tissues processed using any of

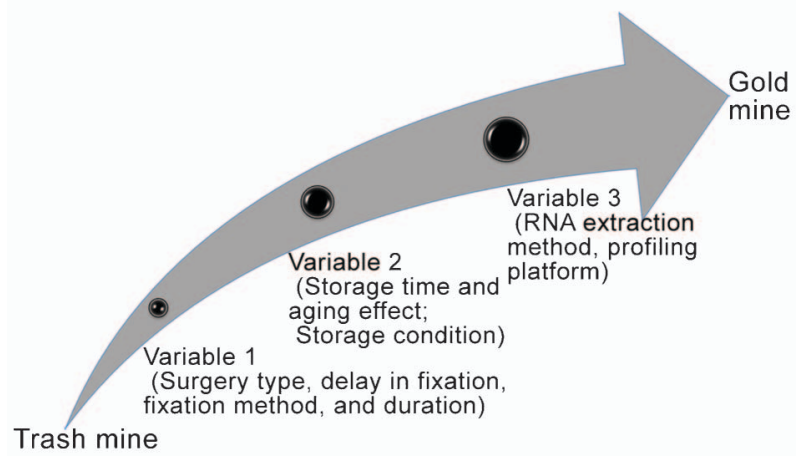

Figure 1 Multiple variables before pathological evaluation (variable 1), after pathological evaluation (variable 2), or at the time of molecular analysis (variable 3), may adversely affect fidelity of molecular profiling data from FFPE specimens. The collection of FFPE specimens can be a gold mine if each of these variables is addressed prior to genome-wide analysis. FFPE, formalin-fixed paraffin-embedded. 


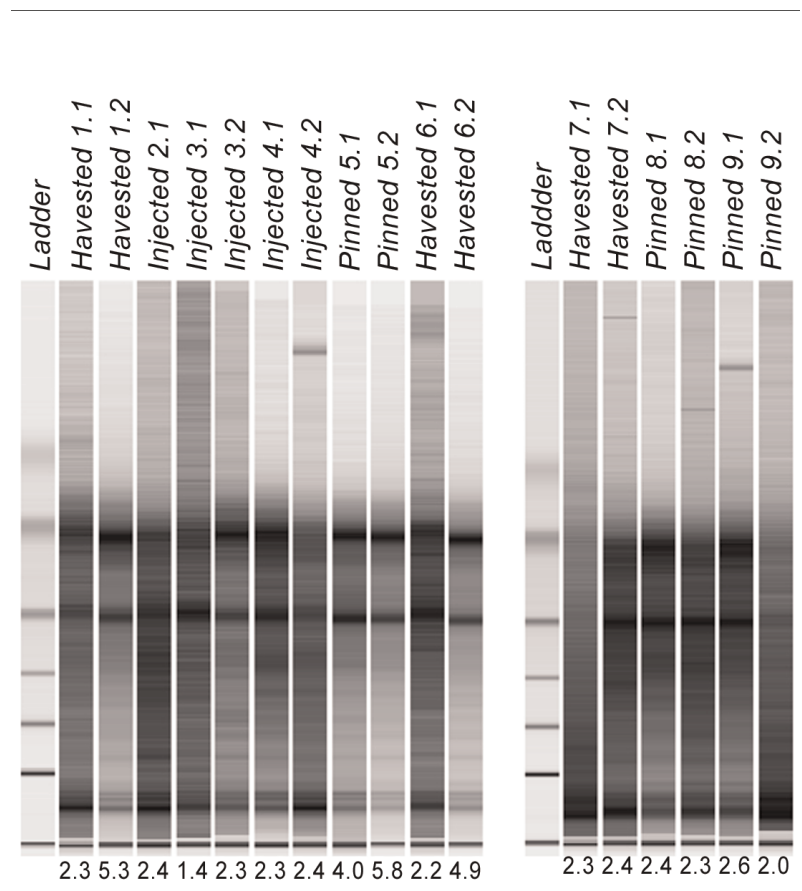

Figure 2 Agilent bioanalyzer electropherograms of 17 total RNA samples from FFPE specimens processed using three different formalin fixation methods employed in the surgical pathology lab at the Johns Hopkins University. Harvested: tissue slices were processed for FFPE after standard tissue-harvesting procedure, during which areas of cancer were cut in duplicate for fresh freezing and FFPE processing in the surgical pathology lab; pinned: tissue slices were processed for FFPE after punch cores of cancer tissue were taken while pinned down; injected: whole-prostate fixation by formalin injection and microwaving. RIN numbers associated with each sample were provided below the corresponding electropherogram (reproduced from Ref. 32). FFPE, formalinfixed paraffin-embedded; RIN, RNA integrity number.

the three formalin fixation methods. We have concluded that highquality total RNA (as evidenced by $28 \mathrm{~S}$ and $18 \mathrm{~S}$ ribosomal RNA bands) can be extracted using the Paradise system (Applied Biosystems, Carlsbad, CA, USA) from FFPE tissues processed in our institution. ${ }^{32}$

\section{HIGH-FIDELITY GENOME-WIDE EXPRESSION DATA DERIVED FROM FFPE TISSUE}

To more rigorously test the reliability of array data from FFPE tissues, we prepared a frozen benign prostatic hyperplasia $(\mathrm{BPH}) /$ tumor $(\mathrm{B} / \mathrm{T})$ pair by the immediate freezing of a palpable prostate tumor and $\mathrm{BPH}$ tissues, and a FFPE B/T pair by the routine FFPE procedure for the corresponding areas immediately adjacent to the harvested tissues. Following two rounds of RNA amplification, expression profiling was performed using the Agilent 60-mer whole-genome array to determine whether expression differences between $\mathrm{BPH}$ and tumor detected by frozen tissues are similar to those detected by the FFPE tissues. Using a two-color design in which $\mathrm{BPH}$ and tumor were directly compared in each array experiment, we generated eight sets of ratios (from eight arrays), four replicates each for the frozen $\mathrm{B} / \mathrm{T}$ profile and FFPE B/T profile (Figure 3). In two of the four replicates, we flipped the dye (dye swap) to assess potential dye bias. Expression ratios were extracted from 8650 genes meeting the single-quality filtration criterion of average signal intensity greater than 1000 . As shown in Figure 4, reproduced from a recent publication, ${ }^{32}$ gene expression data derived from the FFPE B/T pair showed remarkable concordance, both within the replicates and when compared with the

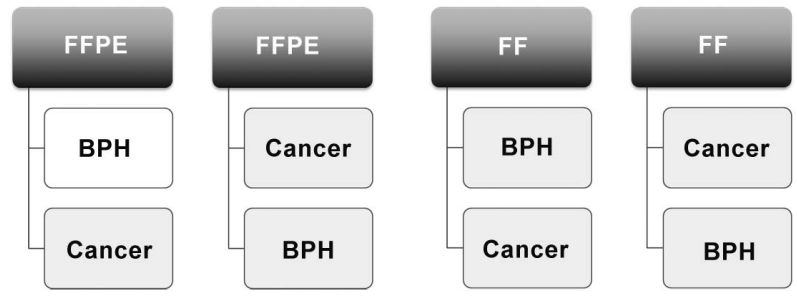

Figure 3 Experiments designed to evaluate genome-wide expression data fidelity using FFPE and frozen tissues processed in parallel. Profiling was performed using the Agilent two-color design with technical replicates $(n=8)$. See Ref. 32 for details. BPH, benign-prostatic hyperplasia; FF, fresh frozen; FFPE, formalinfixed paraffin-embedded.

data derived the frozen B/T pair. As visually evident in Figure $4 a{ }^{32}$ reliable detection of expression difference in FFPE tissues can be achieved across a wide range of fold expression changes, when compared with the frozen tissue standard. Figure $4 b^{32}$ summarizes the general concordance between the median (of the four replicates) ratio of the frozen pair and FFPE pair. Based on four replicated sets of ratios derived from the frozen pair, we estimated the variance of the median ratio for each gene at 0.043 . This translates to an estimation of 1.29-fold deviation from the median ratio at the $99 \%$ confidence interval. In other words, if another four sets of ratios were to be derived from the same frozen pair, we expect $99 \%$ of the genes would deviate from the median ratio by less than 1.29-fold (red lines, Figure $4 \mathbf{b}$ ). ${ }^{32}$ As shown in the scatter plot (Figure $4 \mathbf{b})^{32}$ of the median ratio derived from FFPE tissues against the median ratio from the frozen tissues, $20.6 \%$ of the 8650 genes showed significant deviation (greater than 1.29-fold). Therefore, expression profiling using FFPE tissues resulted in a $79.4 \%$ concordance rate when compared with frozen tissues. It is worth noting that the tissue specimens for freezing and FFPE processing were not strictly identical, and therefore, biological difference (as opposed to FFPE processing) accounted for an unknown percentage of discordance.

Based on these findings, we proposed the concept of 'prospective molecular archiving, ${ }^{32}$ which involves long-term storage of FFPE specimens in conditions compatible with RNA and DNA preservation (e.g., -20 or $-80{ }^{\circ} \mathrm{C}$ ) shortly after the FFPE procedure to avoid further degradation in ambient temperature. This approach will allow the samples to be profiled in the future with minimal adverse effects on RNA and DNA quality and yield and in the meantime streamline the sample acquisition process and establish archival specimens representative of the patient population.

\section{DURATION OF FIXATION MAY BE CRITICALLY IMPORTANT}

To evaluate the possible effect of formalin treatment time on RNA quality, we assessed quality of RNA extracted from in vitro cultured cell pellets subjected to varying length of formalin fixation ranging from 1 min to $\sim 16$ h (overnight fixation). We observed a surprising pattern of RNA degradation that appeared to be inversely correlated with length of formalin fixation (Figure 5). In two independently repeated experiments, severe RNA degradation was observed in cell pellets subjected to shorter formalin exposure, whereas longer formalin fixation time preserves RNA quality. These results suggest to us that under-fixed specimens are not compatible with extraction of highquality RNA. To exclude the possibility that the fixation time effect may be a cell line artifact, we expanded this study to rat prostates subjected to different formalin fixation times. The original observation in cell pellets was largely confirmed in rat prostate tissues, as evident from the higher RNA quality and yield in tissues subjected to longer 

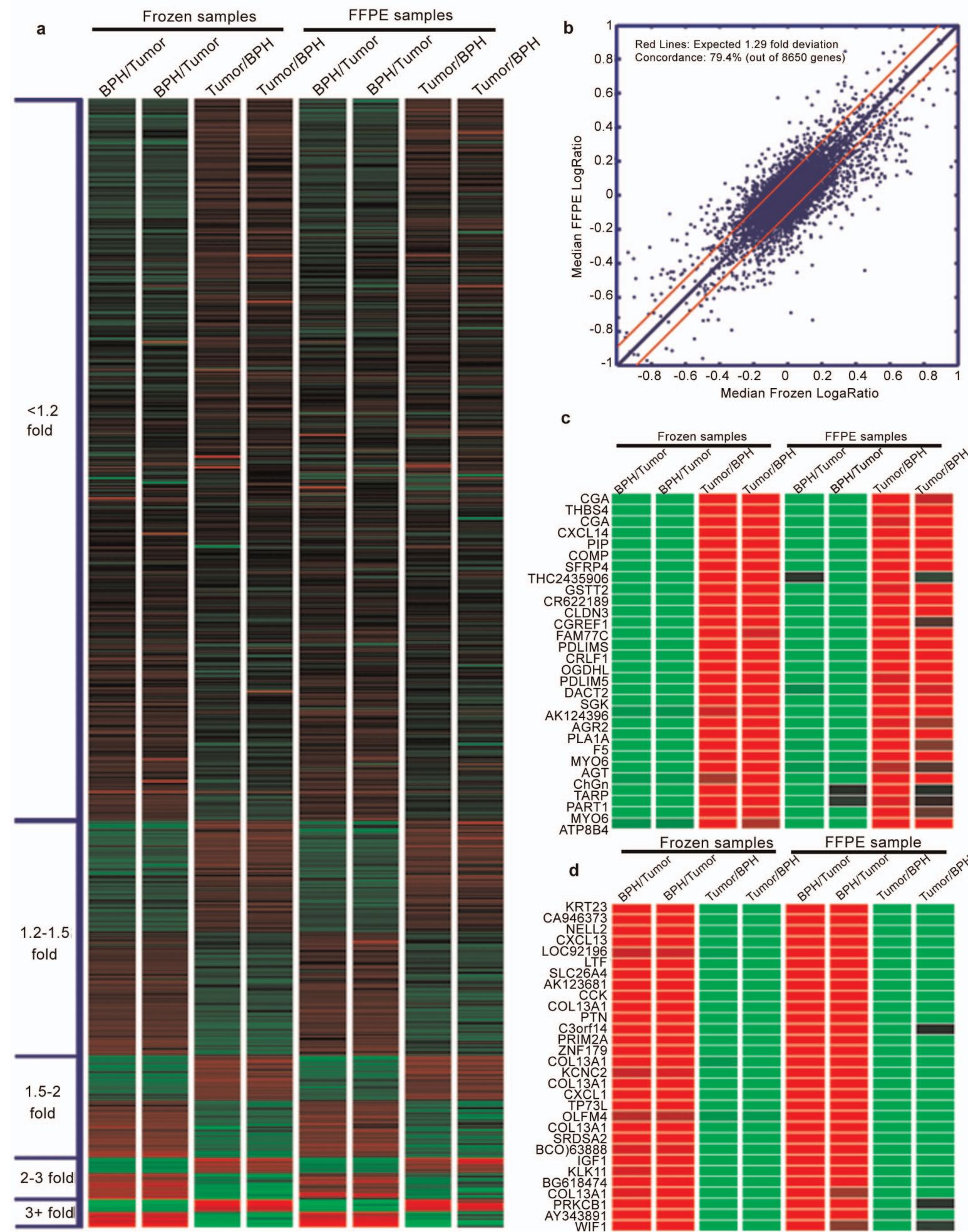

$$
\text { c }
$$
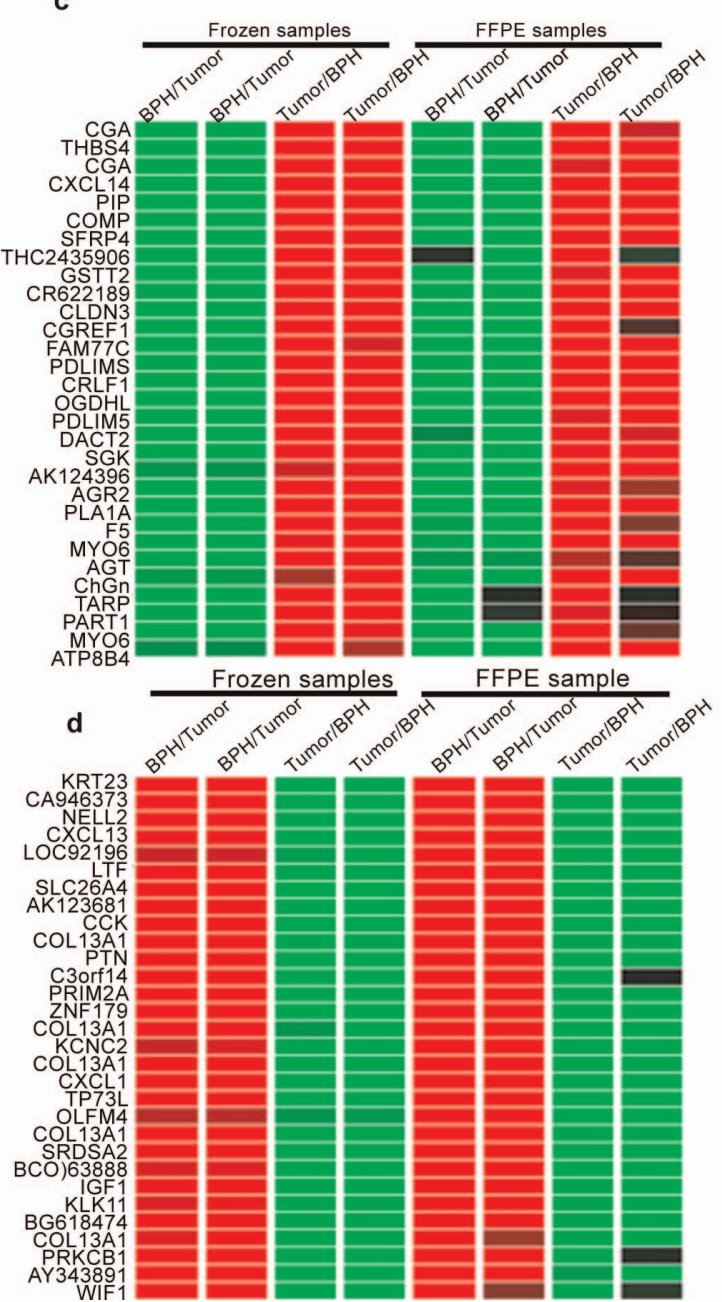

Figure 4 High-fidelity genome-wide expression data from FFPE tissues as assessed by comparing to those from frozen specimens processed in parallel (reproduced from Ref. 32). (a) Heatmap of the 8650 genes ranked (in ascending order) based on median fold expression change between BPH and prostate cancer. Each column represents one of the eight ratios derived from the annotated comparisons. (b) Scatter plot of median expression ratios from FFPE tissues against median ratios from frozen tissues. Red lines denote the expected 1.29-fold deviation at $P<0.01$. (c) Parallel comparison (FFPE vs. frozen) of top ranked genes overexpressed in prostate cancer vs. BPH. (d) Parallel comparison (FFPE vs. frozen) of top 25 genes overexpressed in BPH vs. prostate cancer. BPH, benign prostatic hyperplasia; FFPE, formalin-fixed paraffin-embedded. 


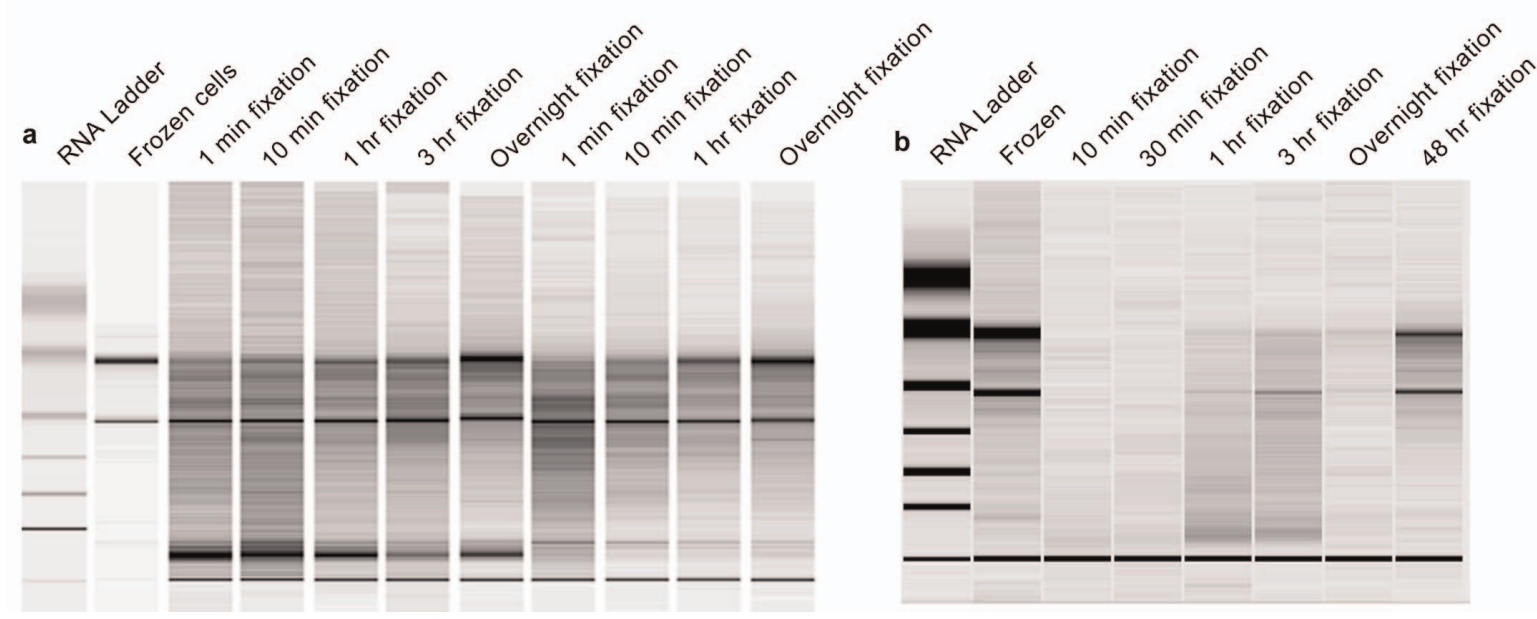

Figure 5 Profound effect of formalin fixation duration on RNA yield and quality in cell pellets (a) and rat prostate tissues (b).

hours of formalin fixation. Thus, the FFPE procedure alone may not be detrimental to RNA or DNA quality, if the fixation duration is optimized and further degradation during long-term storage is controlled.

\section{GENOME-WIDE ANALYSIS OF HIGH-GRADE PROSTATIC INTRAEPITHELIAL NEOPLASIA (HGPIN) LESIONS FROM FFPE SPECIMENS}

We recently performed a pilot study to investigate the expression profile of HGPIN using the Agilent whole-genome expression microarrays. HGPIN may be the precursor tissue lesion to invasive prostate cancer. Molecular analysis of HGPIN has the potential to identify molecular alterations responsible for the transition from in situ neoplasia to invasive cancer. To perform expression analysis of HGPIN lesions, it is necessary to use FFPE tissues compatible with standard histological diagnosis for reliable separation of HGPIN lesions, as this lesion is very difficult to identify in frozen specimens. We performed LCM and dissected four prostate lesions ( $\sim 2000$ cells) representing three HGPIN lesions and one Gleason grade 3 prostate cancer tissue, from four different FFPE sections. Total RNA was extracted and processed for quality control checks. Two rounds of RNA amplification and labeling were performed prior to hybridization onto the Agilent 4X44K whole-genome expression microarrays. A benign FFPE sample similarly processed was used as a common reference in four microarray hybridizations, each with a test sample of either prostate cancer (one sample) or HGPIN (three samples). Expression ratios of test/reference were analyzed. Ratios greater than 1 indicate higher expression in the test sample (cancer or HGPIN) relative to the benign sample and were represented by scaled red color intensities, whereas ratios less than 1 indicate lower expression in the test samples (cancer or HGPIN) relative to the benign sample and were represented by scaled green color intensities.

An important technical indicator of the expression microarray output is the scatter plot of raw intensities from the two channels (Figure 6, $\mathrm{Y}$ axis is the test sample and $\mathrm{X}$ axis is the reference sample). All four microarrays yielded satisfactory results as judged by the distribution of the raw intensities and ratios. The normalized expression ratios were further analyzed. Unsupervised clustering analysis using Pearson's correlation as the similarity measure was performed to assess the overall similarity of the global profiles among the four samples (one cancer and three HGPIN). As shown in Figure 7a, HGPIN samples can be discerned from the cancer sample. In addition, appreciable heterogeneity among the three HGPIN samples was observed. Next, we performed supervised analysis and identified the top genes, by using a weighted gene metric as described in our previous study, ${ }^{33}$ which are differentially expressed between the HGPIN samples and the lone cancer sample. A total of 73 annotated genes are shown in Figure $\mathbf{7 b}$. Among the 73 genes, 44 were higher in cancer while 29 were higher in HGPIN.

It is important to note that caution should be exercised when interpreting the pilot data, owing to their preliminary nature and the very limited number of samples profiled. Nevertheless, this preliminary analysis uncovered potentially interesting differences between HGPIN and prostate cancer. We would like to highlight two findings. First, the expression profiling of HGPIN that was previously reported used dissected frozen specimens, though it is difficult to ascertain HGPIN lesions in frozen specimens. ${ }^{34}$ In that study, 35 annotated genes demonstrated upregulation in transition from HGPIN to cancer. Among these, lumican (LUM) and fibrinogen-like 1 (FGL1) (with * in Figure 7b) were among the 44 genes that are higher in cancer than HGPIN. These genes can be pursued as tissue invasion markers as invasive morphology separates cancer from HGPIN. Second, prostate cancer stem cell antigen (PSCA) was reported as a marker for HGPIN that can be used to predict cancer in repeat biopsies. ${ }^{35}$ PSCA (with * in Figure $7 \mathbf{b}$ ) is highly overexpressed in all three HGPIN lesions relative to the benign sample or the cancer sample. This study, though preliminary, suggest that important biological differences do exist between HGPIN lesions and Gleason grade 3 tumors, which cannot be readily distinguished by morphological differences on the individual cell level. Since the technical aspects of isolating indolent prostate cancer by LCM from FFPE tissues are identical to those involved in this pilot HGPIN study, we also demonstrate the feasibility of genome-wide expression analysis focusing on indolent prostate cancer.

\section{SUMMARY}

Previously, large-scale molecular profiling studies (including our own studies) are inevitably biased toward a subpopulation of prostate cancer patients with large tumor volume and high-risk cancer. To better understand the natural history of prostate cancer progression and address the contemporary problem of prostate cancer overtreatment, it is desired to employ genome-wide approaches to profile prostate cancers that include those representing indolent prostate cancer defined in the setting of active surveillance. Such efforts will entail the establishment of an 

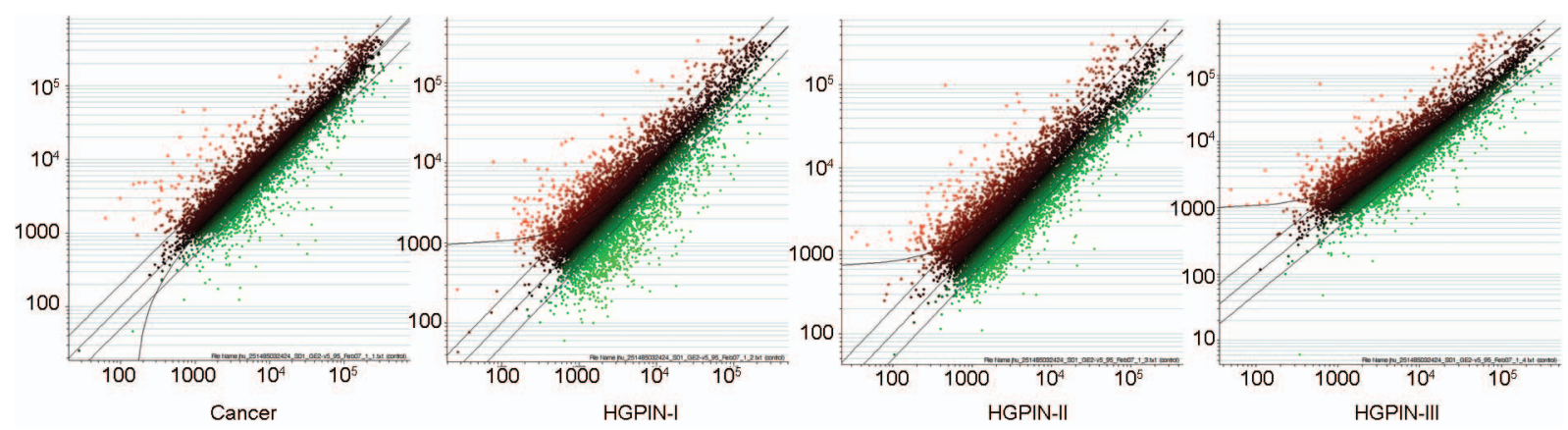

Figure 6 Scatter plots of raw intensities from each of the four microarrays comparing either cancer $(n=1)$ or HGPIN $(n=3)$ tissues to $\mathrm{BPH}$. BPH, benign prostatic hyperplasia; HGPIN, high-grade prostatic intraepithelial neoplasia.

informative cohort of patients diagnosed with very low-risk prostate cancer and followed-up for development of higher-risk disease, and will rely on the quality and quantity of RNA and DNA that can be retrieved from FFPE tissues. Technical hurdles presented by the FFPE specimens in these studies may be overcome by rigorous quality control measures. This is exemplified by our efforts presented here, as well as many other a

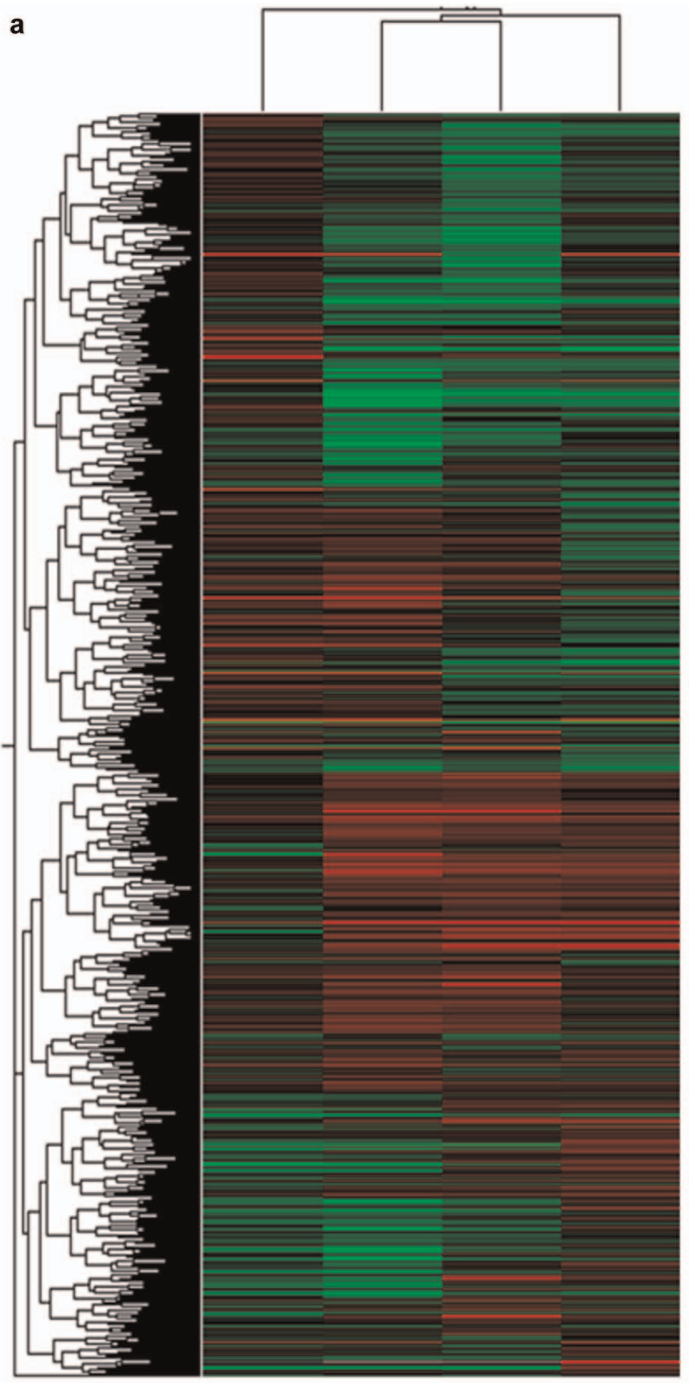

Cancer HGPIN-I HGPIN-II HGPIN-III b

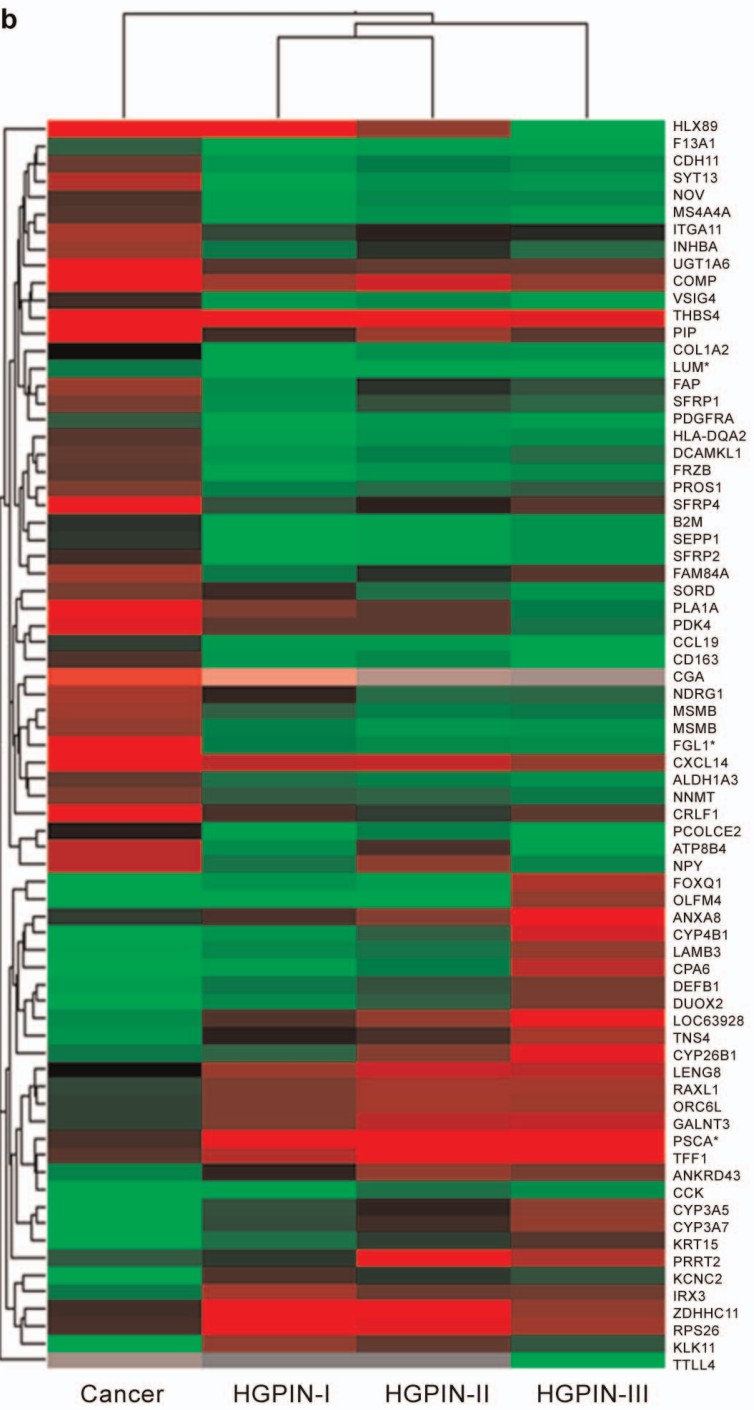

Cancer HGPIN-I HGPIN-II HGPIN-III

Figure 7 Expression ratio analysis of HGPIN. (a) Dendrogram and heatmap of genes and samples following unsupervised clustering analysis of the three HGPIN samples and one cancer sample. (b) Seventy-three genes representing putative candidates differentially expressed between HGPIN and cancer (color intensity reaches saturation at 10 -fold). HGPIN, high-grade prostatic intraepithelial neoplasia. 
recent gene expression studies ${ }^{27-31}$ focusing on expression markers associated with Gleason grade or risk for biochemical recurrence following surgical treatment. These studies, combined with our detailed technical analysis, support the technical feasibility for genome-wide molecular profiling of small volume, potentially indolent prostate cancer. Such studies have not been systemically performed, but represent an urgent priority due to the need to understand indolent prostate cancer, and to develop molecular profiles/markers that define indolent prostate cancer so that men who could safely forego treatment can be effectively identified at the time of diagnosis.

\section{COMPETING FINANCIAL INTERESTS}

The authors declare no competing financial interests.

\section{ACKNOWLEDGMENTS}

The authors would like to thank Gwen Lagoda and for assistance in preparation of the tissue specimens. Some of the work described in this article was supported by the US National Institute of Health grant R21CA106425 (to JL) and the NIH/NCI Specialized Program in Research Excellence in Prostate Cancer grant P50CA58286 (Johns Hopkins University).

1 Tosoian JJ, Trock BJ, Landis P, Feng Z, Epstein JI et al. Active surveillance program for prostate cancer: an update of the Johns Hopkins experience. J Clin Oncol 2011; 29: 2185-90.

2 Klotz L, Zhang L, Lam A, Nam R, Mamedov A et al. Clinical results of long-term followup of a large, active surveillance cohort with localized prostate cancer. J Clin Oncol $2011 ; 28: 126-31$

3 Jemal A, Murray T, Ward E, Samuels A, Tiwari RC et al. Cancer statistics, 2005. CA Cancer J Clin 2005; 55: 10-30.

4 Brenner $\mathrm{H}$. Long-term survival rates of cancer patients achieved by the end of the 20 th century: a period analysis. Lancet 2002; 360: 1131-5.

5 Schroder FH, Hugosson J, Roobol MJ, Tammela TL, Ciatto S et al. Screening and prostate-cancer mortality in a randomized European study. N Engl J Med 2009; 360: 1320-8.

6 Sakr WA, Grignon DJ, Crissman JD, Heilbrun LK, Cassin BJ et al. High grade prostatic intraepithelial neoplasia (HGPIN) and prostatic adenocarcinoma between the ages of 20-69: an autopsy study of 249 cases. In Vivo 1994; 8: 439-43.

7 Powell IJ, Bock CH, Ruterbusch JJ, Sakr W. Evidence supports a faster growth rate and/or earlier transformation to clinically significant prostate cancer in black than in white American men, and influences racial progression and mortality disparity. J Uro 2011; 183: 1792-6.

8 Etzioni R, Penson DF, Legler JM, di Tommaso D, Boer R et al. Overdiagnosis due to prostate-specific antigen screening: lessons from U.S. prostate cancer incidence trends. J Natl Cancer Inst 2002; 94: 981-90.

9 Thompson IM, Goodman PJ, Tangen CM, Lucia MS, Miller GJ et al. The influence of finasteride on the development of prostate cancer. N Engl J Med 2003; 349: 215-24.

10 Thaxton CS, Loeb S, Roehl KA, Kan D, Catalona WJ. Treatment outcomes of radical prostatectomy in potential candidates for 3 published active surveillance protocols. Urology 2010; 75: 414-8.

11 Carter HB, Walsh PC, Landis P, Epstein JI. Expectant management of nonpalpable prostate cancer with curative intent: preliminary results. J Urol 2002; 167: 1231-4.
12 Bastian PJ, Yegnasubramanian S, Palapattu GS, Rogers CG, Lin X et al. Molecular biomarker in prostate cancer: the role of CpG island hypermethylation. Eur Urol 2004; 46: 698-708.

13 Warlick C, Trock BJ, Landis P, Epstein JI, Carter HB. Delayed versus immediate surgical intervention and prostate cancer outcome. J Natl Cancer Inst 2006; 98 : 355-7.

14 Carter HB, Ferrucci L, Kettermann A, Landis P, Wright EJ et al. Detection of lifethreatening prostate cancer with prostate-specific antigen velocity during a window of curability. J Nat/ Cancer Inst 2006; 98: 1521-7.

15 Ross AE, Loeb S, Landis P, Partin AW, Epstein Jl et al. Prostate-specific antigen kinetics during follow-up are an unreliable trigger for intervention in a prostate cancer surveillance program. J Clin Oncol 2010; 28: 2810-6.

16 Epstein JI, Walsh PC, Carmichael M, Brendler CB. Pathologic and clinical findings to predict tumor extent of nonpalpable (stage T1c) prostate cancer. JAMA 1994; 271: 368-74.

17 Mufarrij P, Sankin A, Godoy G, Lepor H. Pathologic outcomes of candidates for active surveillance undergoing radical prostatectomy. Urology 2010; 76: 689-92.

18 Ding Z, Wu CJ, Chu GC, Xiao Y, Ho D et al. SMAD4-dependent barrier constrains prostate cancer growth and metastatic progression. Nature 2011; 470: 269-73.

19 Taylor BS, Schultz N, Hieronymus H, Gopalan A, Xiao Y et al. Integrative genomic profiling of human prostate cancer. Cancer Cell 2010; 18: 11-22.

20 Tomlins SA, Mehra R, Rhodes DR, Cao X, Wang L et al. Integrative molecular concept modeling of prostate cancer progression. Nat Genet 2007; 39: 41-51.

21 Bostwick DG, Brawer MK. Prostatic intra-epithelial neoplasia and early invasion in prostate cancer. Cancer 1987; 59: 788-94.

22 Hanahan D, Weinberg RA. The hallmarks of cancer. Cell 2000; 100: 57-70.

23 Perou CM, Sorlie T, Eisen MB, van de Rijn M, Jeffrey SS et al. Molecular portraits of human breast tumours. Nature 2000; 406: 747-52.

24 Liu W, Laitinen S, Khan S, Vihinen M, Kowalski J et al. Copy number analysis indicates monoclonal origin of lethal metastatic prostate cancer. Nat Med 2009; 15: 559-65.

25 Ramaswamy S, Ross KN, Lander ES, Golub TR. A molecular signature of metastasis in primary solid tumors. Nat Genet 2003; 33: 49-54.

26 Hedenfalk I, Duggan D, Chen Y, Radmacher M, Bittner M et al. Gene-expression profiles in hereditary breast cancer. N Engl J Med 2001; 344: 539-48.

27 Nakagawa T, Kollmeyer TM, Morlan BW, Anderson SK, Bergstralh EJ et al. A tissue biomarker panel predicting systemic progression after PSA recurrence post-definitive prostate cancer therapy. PLOS ONE 2008; 3: e2318.

28 Penney KL, Sinnott JA, Fall K, Pawitan Y, Hoshida Y et al. mRNA expression signature of Gleason grade predicts lethal prostate cancer. J Clin Oncol 2011; 29: 2391-6.

29 Ross AE, Marchionni L, Vuica-Ross M, Cheadle C, Fan J et al. Gene expression pathways of high grade localized prostate cancer. Prostate; e-pub ahead of print 25 February 2011; doi:10.1002/pros.21373.

30 Sboner A, Demichelis F, Calza S, Pawitan Y, Setlur SR et al. Molecular sampling of prostate cancer: a dilemma for predicting disease progression. BMC Med Genomics 2010; $3: 8$.

31 Setlur SR, Mertz KD, Hoshida Y, Demichelis F, Lupien M et al. Estrogen-dependent signaling in a molecularly distinct subclass of aggressive prostate cancer. J Natl Cancer Inst 2008; 100: 815-25

32 Dunn TA, Fedor H, Isaacs WB, de Marzo AM, Luo J. Genome-wide expression analysis of recently processed formalin-fixed paraffin embedded human prostate tissues. Prostate 2009; 69: 214-8.

33 Luo J, Duggan DJ, Chen Y, Sauvageot J, Ewing CM et al. Human prostate cancer and benign prostatic hyperplasia: molecular dissection by gene expression profiling. Cancer Res 2001; 61: 4683-8.

34 Ashida S, Nakagawa H, Katagiri T, Furihata M, liizumi M et al. Molecular features of the transition from prostatic intraepithelial neoplasia (PIN) to prostate cancer: genome-wide gene-expression profiles of prostate cancers and PINs. Cancer Res 2004; 64: 5963-72.

35 Zhigang Z, Wenlu S. Prostate stem cell antigen (PSCA) mRNA expression in prostatic intraepithelial neoplasia: implications for the development of prostate cancer. Prostate 2007; 67: 1143-51. 